Introduction

\title{
Redéfinir la littérature francophone : Validation d'un paradigme
}

\author{
Laté Lawson-Hellu
}

\author{
(Western University, Canada)
}

Plus tard, on dira peut-être que ce fut un moment historique : le Goncourt, le Grand prix du roman de l'Académie française, le Renaudot, le Fémina, le Goncourt des lycéens, décernés le même automne à des écrivains d'Outre-France. Simple hasard d'une rentrée éditoriale concentrant par exception les talents venus de la " périphérie », simple détour vagabond avant que le fleuve revienne dans son lit? Nous pensons, au contraire : révolution copernicienne. Copernicienne, parce qu'elle révèle ce que le milieu littéraire savait déjà, sans l'admettre : le centre, ce point depuis lequel était supposée rayonner une littérature franco-française n'est plus le centre. Le centre jusqu'ici, même si de moins en moins, avait eu cette capacité d'absorption qui contraignait les auteurs venus d'ailleurs à se dépouiller de leurs bagages avant de se fondre dans le creuset de la langue et de son histoire nationale : le centre, nous disent les prix d'automne, est désormais partout, aux quatre coins du monde. Fin de la francophonie. Et naissance d'une littérature-monde en français. (...)

(Michel Lebris, Le Monde, du 16 mars 2007)

Vous comprendrez donc que j'applaudisse à deux mains lorsque je lis, dans "Le Monde des livres" (du 16 mars), le brillant hommage de quarante-quatre écrivains à la "littérature-monde" en français ! Nous partageons tous le même éclatant et stimulant constat, à savoir que "diverses sont aujourd'hui les littératures de langue française". Il est clair, aussi, que nous partageons le même objectif, celui "d'un dialogue dans un vaste ensemble polyphonique". Mais vous me permettrez de vous faire irrespectueusement remarquer, mesdames et messieurs les écrivains, que vous contribuez dans ce manifeste, avec toute l'autorité que votre talent confère à votre parole, à entretenir le plus grave des contresens sur la francophonie, en confondant francocentrisme et francophonie, en confondant exception culturelle et diversité culturelle. (...) 
Ce premier numéro de Recherches Francophones, la revue de l'AIELCEF (Association internationale d'étude des littératures et cultures de l'espace francophone), fait suite au colloque international organisé les 7, 8 et 9 juillet 2017 à l'université Western, à London, au Canada, dix ans après le «Manifeste des 44 » qui décrétait la mort de la littérature francophone ( $\mathrm{La}$ « francophonie » dans la déclaration). Ce colloque avait pour thème « Repenser le fait francophone : subjectivités constitutives et reconfiguration épistémologique», et visait à redéfinir, à partir du principe des subjectivités qui le constituent, le fait francophone à la base de l'idée de la littérature francophone. C'est un premier numéro qui permet ainsi, non pas uniquement de valider la portée internationale, régionale et multiple de ce «fait francophone », mais aussi, à un niveau plus historiographique et littéraire, de dépasser le seul critère linguistique officiel de définition de cette littérature. Ce sont alors, à rappeler, tous les aspects distinctifs de ces subjectivités individuelles et collectives qui, dans leur pluralité intrinsèque, constituent une telle littérature et permettent de lui maintenir sa pertinence institutionnelle, historiographique et heuristique, c'est-à-dire à la fois dans sa compréhension historique et dans son appréhension critique, historiographique, tout comme dans son enseignement, où interviennent ses dimensions géographiques, humaines, culturelles, politiques, idéologiques, (socio)linguistiques, institutionnelles, esthétiques, discursives, philosophiques ou épistémologiques d'intelligibilité.

Le dossier du numéro part en effet d'un constat, qui constitue également sa propre pertinence pour le champ de la littérature produite naguère dans l'espace colonisé par l'Europe, et aujourd'hui intégré dans le « Commonwealth » des pays post-colonisés, eux-mêmes intégrés dans le modèle de l'État moderne, la 
pertinence maintenue du paradigme de la Littérature francophone. En 2007, ce paradigme, et la réalité qu'il subsume, est invalidé, et avec lui tout le champ littéraire francophone auquel il renvoyait. Une décennie après, le colloque de 2017 l'ayant prouvé, tout autant que la production continue de travaux portant le particule «francophone ${ }^{1}$, une telle démarche, forte de son fondement institutionnel, a montré ses limites, voire son inadéquation, pour autoriser son questionnement et la recherche de nouveaux modes d'appréhension épistémologique, ce que le colloque de London visait dans ses travaux et propositions épistémiques. Si la littérature francophone existe en tant que champ dans son fonctionnement institutionnel, du point de vue de la recherche, des publications, des colloques, etc., elle démontre une présence, ou une pérennité, qui remet en cause le projet de son éviction. En outre, et au-delà de cette présence qui pose sa question épistémologique et trouve un relais dans l'observation empirique, elle demeure, tant dans ses traits que dans ses réalisations du passé ou du présent. Il fallait donc proposer de nouveaux modes d'appréhension qui permettent de circonscrire une telle littérature devenue témoin de l'histoire de la modernité, celle-là même qui inclut le fait colonial et ses avatars. C'est par le biais de la question de ses subjectivités constitutives qu'il a donc semblé opportun d'entreprendre ce travail de reconfiguration dont fait état le dossier du numéro.

\footnotetext{
${ }^{1}$ On prendra pour exemples parmi d'autres, et pour la seule année 2019, la parution de l'essai d'Alexie Tcheuyap et d'Hervé Tchumkan, Avoir peur. Insécurité et roman en Afrique francophone (2019), et la tenue, à l'Île Maurice, la même année, les 11, 12 et 13 juillet, du colloque international de littérature et linguistique avec pour thème, "Résistance, Résilience, Réactualisation». Le volet littérature de ce colloque portait notamment sur «Les littératures francophones », tel qu'annoncé dès novembre 2018 sur le site Fabula : https ://www.fabula.org/actualites/colloque-international-litterature-et-linguistique-resistanceresilience-reactualisation_86412.php .
} 
Que dit donc la réflexion épistémologique sur la question de la subjectivité ? $\mathrm{Si}$, en effet, pour cette réflexion, l'objectivité, ou le statut de l'objet en dehors de sa perception par un sujet, fait partie de la réalité, la subjectivité, qui renvoie à la perception de la réalité par un sujet, fait moins objet de certitude, d'où tout le débat épistémologique autour de la subjectivité et de ses définitions. Pour le fait francophone, quelle serait ainsi la «subjectivité » à partir de laquelle proposer une redéfinition du fait littéraire, lorsque la question se pose comme dans le cadre de ce numéro des Recherches Francophones, mais aussi dans celui des problèmes institutionnels, et donc épistémologiques aussi, du fait francophone et du fait littéraire francophone en soi, du point de vue multiple de leur histoire liée au fait colonial européen? Pour la réflexion épistémologique sur la question de l'objectivité, il est entendu, également, que l'utilisation des termes « objectivité » et « subjectivité » renvoie aux différences de sens entre la « réalité objective » et les «impressions subjectives ». Il s'agit, pour nous, de découvrir la validité, épistémologique, ici aussi, du choix du paradigme de la subjectivité pour cette autre appréhension à proposer du fait francophone, et, ainsi, du fait littéraire francophone en soi. L'article de Dwayne H. Mulder [s. d.], que nous retenons ici, sur la question de l'objectivité, «Objectivity (IEP, Internet Encyclopedia of Philosophy), permet de suivre brièvement le débat philosophique sur une telle question et d'en déduire la pertinence pour la question littéraire francophone.

Pour cet article, en effet, l'utilisation philosophique des deux termes de la subjectivité et de l'objectivité débouche sur la distinction entre ce qui serait la connaissance objective et la connaissance subjective. Si la première traite de l'objet supposé exister en dehors de son appréhension par une conscience 
subjective, la seconde renvoie à tout aspect de la réalité dont l'appréhension repose foncièrement sur une conscience subjective: les sentiments ou la perception sensorielle, celle des couleurs, par exemple, de leur « existence ». La connaissance objective évoque donc une réalité objective alors que la connaissance subjective, une réalité subjective ainsi définie. En épistémologie, qui est aussi l'étude philosophique de la connaissance, comme l'indique l'article de D. H. Mulder, la distinction précise que la connaissance subjective relève davantage de la connaissance provenant de l'état subjectif du sujet de la connaissance. Pour le propos, ici, cette distinction ouvre ainsi la voie aux conditions d'ensemble de cet état où interviennent par exemple des principes comme l'idéologie, les systèmes de valeurs, les références imagologiques du sujet, autrement dit, son rapport à la réalité et aux autres, la formulation minimale de la subjectivité. Pour la réflexion épistémologique encore, la question de la connaissance objective soulève plutôt celle de sa base de validation. Ici, également, s'élargit le débat, pour intégrer à la fois les critères de validation de ce qui est la connaissance objective au point d'inférer son invalidité devant le relais nécessaire que constitue la conscience subjective dans l'appréhension de tout aspect de la réalité. En d'autres termes, et c'est ce qui donne sens à toute la branche philosophique de la phénoménologie, c'est la nécessité de recourir à la perception subjective de l'individu, au travail de son cerveau, dans le rapport cognitif à la réalité, qui entraine, alors, la possibilité d'invalidation de toute connaissance « objective ». Au nombre, cependant, des critères de validation de la connaissance objective, figurent, au fil de l'histoire de la pensée philosophique en Occident, l'intersubjectivité, où le consensus formé par deux ou plusieurs appréhensions subjectives peut fonder une connaissance objective, ou encore, tel au XVII ${ }^{\mathrm{e}}$ siècle français, la foi dans la raison. La rationalité, même de l'individualité subjective, devient ici la base de l'objectivité de son appréhension 
de la réalité. Dans la pensée phénoménologique, c'est l'impossibilité à la conscience de l'individu d'intégrer la réalité de l'objet soumis à son appréhension qui aura conduit à la préférence de la connaissance subjective en lieu et place de l'impossibilité épistémologique de la connaissance objective. Dans la question de la littérature francophone et du fait francophone, deux réalités intrinsèquement liées aux subjectivités individuelles et collectives locales soumises à la perception étrangère, coloniale naguère, la question de la connaissance subjective prend toute son importance devant la connaissance «objective » qui en a été proposée dans la propre implication idéologique, et donc « subjective », du sujet de l'appréhension « objective ». Si le fait francophone a été défini par le fait de la langue, une telle connaissance a été jugée objective et donc a pu donner lieu à des décisions institutionnelles qui vont du critère exclusif de la langue à l'instauration de cadres institutionnels reposant sur un tel critère de la langue.

Dans son introduction au numéro spécial de la revue Discours social / Social Discourse, «Rethinking the subject in discourse: From relative subjectivity to the ontology of subject as related interiority » (Marike Finlay, 1989), notamment sur le problème de la conception du sujet dans la théorie sociale postmoderne, Marike Finlay aborde une telle perspective de la question de la subjectivité. On remarquera, toutefois, que l'approche ainsi proposée de cette question de la subjectivité s'inscrit dans la perspective psychanalytique d'un projet d'analyse discursive de la problématique du « sujet ». Si, donc, comme le propose M. Finlay, la question de la subjectivité est au cœur de la réflexion philosophique occidentale tout au long du $\mathrm{XX}^{\mathrm{e}}$ siècle, elle repose sur l'assertion que la modernité a théoriquement construit un sujet doué de raison et de pouvoir, et que la réflexion post-moderne du dernier tiers du siècle a révoqué ce 
«pouvoir» constituant du sujet. Le sujet, dans sa dimension susceptible d'échapper à la préhension de la réflexion philosophique, sa dimension « ontologique », tend ainsi à être mis de côté au profit des crises socio-politiques, économiques et idéologiques du monde occidental, des crises définies ellesmêmes par les rivalités historiques internes à l'Europe des siècles précédents puis de l'ensemble du monde occidental. Définir la subjectivité de l'individu, d'où qu'il vienne sur la planète, demande dès lors de prendre en considération ce qui en fait un être «vivant», et dont les principes constitutifs excèdent sa propre appréhension. Ce qui en reste demeure l'affectation qu'en produisent ses conditions multiples d'existence, du système culturel au système idéologique. C'est le noyau épistémique de la réflexion de Marike Finlay. La prise de conscience du sujet sur son existence et sa constitution en tant que tel peut être débattue, quant à son moment d'émergence, question qu'il ne faudrait pas dissocier des faits de culture dans lesquels le sujet arrive à cette conscience, mais cette prise de conscience demeure assujettie aux conditions socio-culturelles de son expression. C'est à ce niveau de l'expression de la subjectivité qu'intervient la pratique artistique, dont le fait littéraire. Si le moment exact de la prise de conscience de la subjectivité peut faire débat, le moment de l'expression de cette subjectivité le fait moins, puisque l'expression artistique va témoigner de la combinaison des intrants, et de la « personnalité » et de la part insaisissable (donc «ontologique ») du sujet, qui deviennent la mesure de sa subjectivité. L'observateur ou l'analyste est alors en mesure d'en faire état et d'en reconstituer la part observable, voire quantifiable. La réflexion de Marike Finlay y revient, mais à partir de la question de la théorie sociale et de son incidence, notamment le problème de la dépersonnalisation du sujet, sur la conceptualisation de la subjectivité sociale de l'individu. Il est question, chez elle, d'une approche plutôt pragmatique de la question de la subjectivité, qui n'évacue donc pas la part 
«socio-idéologique et discursive» nécessaire de son modelage et, pour l'analyste, de son appréhension épistémologique. Une telle approche quitte ainsi le domaine de la spéculation pour offrir des bases d'appréhension ensuite conceptualisables, c'est-à-dire définissables. La même conception serait alors applicable au statut de sujet de l'écrivain francophone ${ }^{2}$. La question du sujet francophone, du point de vue institutionnel de la pratique littéraire francophone, devient de ce fait une question formulable dont les éléments de réponse seraient également formulables, et des propositions de définition antérieures, susceptibles d'invalidation, de contestation, sinon de confirmation ou de reconfiguration. De ce point de vue pourrait se formuler ainsi la reconfiguration du fait littéraire francophone, de la littérature francophone, à partir de ses subjectivités constitutives, c'est-à-dire de ses individus producteurs aux collectivités dont se réclament ces individus.

Sur la prévalence épistémologique de la question subjectiviste, celle notamment de la connaissance subjective, que la réflexion, ici, privilégie comme cadre d'appréhension du fait francophone, et donc du fait littéraire francophone, on remarquera aussi que la littérature francophone, pour l'institution nationale qui en bénéficie, renvoie à la difficulté épistémologique déjà évoquée de la connaissance objective. $\mathrm{Si}$, sur ce plan philosophique, que nous privilégions, comme il s'agit d'une reconfiguration proposée de ce fait institutionnel dans les problèmes de fond de ses appréhensions d'usage, la connaissance subjective, ou le biais de la connaissance subjective de son appréhension, constitue un contre-

\footnotetext{
${ }^{2}$ Il ne s'agit alors plus de se limiter par exemple à l'histoire coloniale dans cette appréhension de la subjectivité de l'individu francophone, comme le propose notamment Bodia Bavuidi dans son ouvrage, Subjectivités et écritures de la diaspora francophone. Maryse Condé, Alain Mabanckou et Melchior Mbonimpa (2015).
} 
poids à de tels problèmes de fond, bases de la connaissance objective supposée en être la validation. Ici aussi, la réflexion rappelle tout l'investissement individuel, collectif, historique, politique, idéologique, voire stratégique, hier et aujourd'hui, autour de cette connaissance objective du fait francophone. Le critère de la langue, qui lui est retenu, en dit toute la mesure. C'est donc sur la base de la connaissance subjective de la réalité, telle que l'offrent les individualités constitutives du fait francophone, c'est-à-dire à ce niveau épistémologique, que devrait se situer la validité du paradigme de la subjectivité comme critère d'appréhension de ce fait institutionnel, mais aussi identitaire et collectif rassemblé sous la catégorie, devenue idéologique, de la francophonie. Les articles rassemblés dans le numéro en donnent une mesure, en explorant des pistes de mise en exergue des instances de subjectivités locales, nécessaires, à la base d'intelligibilité, épistémologique, en somme, du fait francophone.

Le dossier du numéro s'organise ainsi en trois sections consacrées à la perspective épistémologique, à la perspective linguistique et à la perspective ontologique de ces exemples d'expressions de subjectivités francophones à pouvoir prendre en considération dans tout projet, comme celui du numéro et de son colloque international de référence, de reconfiguration épistémologique du fait francophone et de l'expression littéraire francophone. Si la perspective épistémologique de cette question des subjectivités francophones présente, dans les articles qui y sont rassemblés, une réflexion sur la propre question de la subjectivité, et présente des cas d'étude ou d'expressions de la subjectivité en contexte problématique, voire hégémonique, la perspective linguistique, dans les articles qui y sont rassemblés, propose une approche théorique de la question de la subjectivité, mais dans une perspective linguistique de l'énonciation, avec des 
cas de problématisation de telle subjectivité. La troisième section du dossier, la perspective ontologique, à travers les articles qui y sont rassemblés, propose une approche de la question de la subjectivité dans une perspective épistémologique, certes, mais qui privilégie la part ontologique du sujet, son agentivité, pour le formuler ainsi. Cinq articles figurent dans la première section, ceux d'Andrea Bellia, de François Paré, d'Hervé Ondoua, de Laté Lawson-Hellu et de Samir Messaoudi. Quatre articles figurent dans la deuxième section, ceux d'Amidou Sanogo, de Louis Ndong, de Judith Sinanga-Ohlmann et de Fida Dakroub. Deux articles figurent dans la troisième section, ceux de Mbaye Diouf et de Hassan Moustir.

Dans son article, Andrea Bellia présente, dans la ligne de la pensée de Descartes et à travers une lecture de Marcel Proust, la philosophie réflexive, c'est-à-dire le fondement métaphysique du moi au-delà de son réel psychologique. L'article de François Paré présente, pour sa part, une réflexion sur la problématique de la langue maternelle, ou première, dans un contexte historique de domination (coloniale), mais aussi de résistance du sujet (colonisé), tel Abdelkébir Khatibi, auteur marocain du XX $X^{\mathrm{e}}$ siècle. L'article d'Hervé Ondoua propose la critique de la rationalité occidentale dans la pensée « subalterne » de Gayatri Spivak, particulièrement dans une perspective postcoloniale de construction nationale, c'est-à-dire anticolonialiste. L'article de Laté LawsonHellu présente les expressions du paradigme de la question de la terre dans des exemples d'œuvres produites dans des cas de cultures marquées par l'histoire coloniale, notamment les cultures kanak, du Pacifique, et guin-ewe, de l'Afrique subsaharienne. Dans ces cas, l'écriture devient une modalité de résistance à l'histoire coloniale. L'article de Samir Messaoudi étudie un cas d'écriture de la 
situation du sujet colonisé devant le poids du pouvoir sur sa subjectivité d'individu et d'écrivain, dans une démarche réflexive qui devient alors philosophique, et qui pose, en somme, la question de la subjectivité de l'écriture.

Dans la section consacrée à la perspective linguistique, l'article d'Amidou Sanogo propose une réflexion sur la subjectivité et sa représentation en linguistique, notamment dans le cas particulier de la subjectivité francophone. L'article de Louis Ndong étudie, pour sa part, le cas inusité de l'écriture romanesque, en français, qui fait suite, chez Ousmane Sembène, écrivain et cinéaste, à la version cinématographique de la même œuvre produite en wolof, cependant, langue locale. Son interrogation subséquente porte sur les enjeux ainsi soulevés par ce recours, à rebours, au français dans un contexte initial d'expression artistique non-francophone. L'article de Judith Sinanga-Ohlmann étudie plutôt les conditions épistémologiques de l'appropriation de la langue française chez les auteurs francophones d'Afrique subsaharienne, avec une lecture de romans tirés du corpus francophone africain subsaharien. L'article de Fida Dakroub propose, dans le même cadre épistémologique, une lecture de la réflexion d'Amin Maalouf placée dans le cadre de l'expression de la subjectivité linguistique francophone au Liban, mais en termes de stratégie identitaire.

Dans la troisème section, consacrée à la perspective ontologique, l'article de Mbaye Diouf propose de relire les « subjectivités senghoriennes » dans ce qui serait alors une perspective du «Care», ou du «Soin», c'est-à-dire de sollicitude herméneutique, dans des cadres de crises de la réalité telle l'actualité pandémique de ce début du $\mathrm{XXI}^{\mathrm{e}}$ siècle, mais aussi dans le contexte du temps de Senghor, au Sénégal, dans le courant du $\mathrm{XX}^{\mathrm{e}}$ siècle. L'article de Hassan Moustir 
présente, enfin, dans le sens de la prévalence des subjectivités, individuelles ou collectives, francophones, le processus d'autonomisation de la littérature marocaine de langue française, par-delà la référence historique et culturelle nationale.

Dans l'ensemble de ces articles rassemblés dans le dossier, revient la question cruciale du rapport du fait francophone à ses cadres historiques d'intelligibilité, le contexte colonial et, dans une moindre mesure, le contexte national post-colonial. Dans chacun de ces articles, et c'est ce qui donne alors une idée de la question des subjectivités francophones à prendre en considération désormais, revient la question, également cruciale, ontologique, nécessairement, de la résistance du sujet. L'un des objectifs du colloque d'origine était d'arriver à proposer une piste d'appréhension autre du fait francophone et de son expression littéraire par le biais de leurs subjectivités constitutives. L'intuition, déjà existante avant ce colloque, ne serait-ce que dans le paradigme de la diversité que le discours institutionnel adjoint souvent à la prééminance de sa langue de référence, le français, d'où son paradigme premier de l'unité, se confirme ici, de la validité du paradigme de la résistance à inscrire alors dans la question des subjectivités francophones, ou dans l'intelligibilité épistémique du paradigme de la subjectivité proposé. Les articles du numéro en offrent un aperçu. 


\section{Bibliographie}

Bavuidi, Bodia, Subjectivités et écritures de la diaspora francophone. Maryse Condé, Alain Mabanckou et Melchior Mbonimpa, Paris, L'Harmattan, 2015.

Finlay, Marike, « Rethinking the subject in discourse : From relative subjectivity to the ontology of subject as related interiority », Discours social / Social Discourse, Volume II, ${ }^{\text {os }} 1 \&$ 2, Spring-Summer 1989, p. ix-liv.

Mulder, Dwayne H., "Objectivity", Internet Encyclopedia of Philosophy, https://iep.utm.edu/object/ [s. d.] [consulté le 8 septembre 2021].

Tcheuyap, Alexie et d'Hervé Tchumkan, Avoir peur. Insécurité et roman en Afrique francophone, Québec, Presses de l'Université Laval, Col. « Littérature et imaginaire contemporain », 2019. 